\title{
Intensive blood-pressure lowering in patients with acute cerebral hemorrhage
}

\author{
Reviewed by: Sufyan Alrahbi, MD*; Rashid Alaraimi, MBChB*; Abdalla Alzaabi, MBBS*; \\ Sophie Gosselin, MD ${ }^{\dagger \neq}$
}

\begin{abstract}
Clinical question
Is intensive blood pressure (BP) treatment (systolic BP target $110-139 \mathrm{~mm} \mathrm{Hg}$ ) better than standard antihypertensive treatment (systolic BP target $140-179 \mathrm{~mm} \mathrm{Hg}$ ) in reducing mortality and disability in patients with acute intracerebral hemorrhage (ICH)?

Article chosen

Qureshi Al, Palesch YY, Barsan WG, et al. Intensive blood-pressure lowering in patients with acute cerebral hemorrhage. N Engl J Med 2016;375(11):1033-43.

Objective

To determine the therapeutic benefit of intensive BP treatment compared to standard BP treatment in reducing death and disability after 3 months of follow-up among patients with $\mathrm{ICH}$ treated within 4.5 hours from onset of symptoms.
\end{abstract}

Keywords: blood pressure, $\mathrm{ICH}$, intensive, intracerebral
hemorrhage, labetalol, nicardipine

\section{BACKGROUND}

Hematoma expansion following acute cerebral bleeding has been postulated to be the reason for increasing morbidity and mortality. ${ }^{1}$ The acute hypertensive response in the first few hours following ICH may increase hematoma size and growth. This resulted in the hypothesis that by rapidly and aggressively lowering the $\mathrm{BP}$, the hematoma expansion will be reduced, thus improving death and disability among patients with $\mathrm{ICH}^{2}$ The Second Intensive Blood Pressure Reduction Trial (INTERACT2) that recruited patients with spontaneous ICH who had BP $(150-220 \mathrm{~mm} \mathrm{Hg})$ within 6 hours of symptoms onset showed no significant reduction in death or disability between the intensive and standard BP treatment groups. However, an ordinal analysis of the modified Rankin score showed a statistically significant favorable shift in the distribution scores of their secondary outcomes. ${ }^{3}$ This second study of the Antihypertensive Treatment of Acute Cerebral Hemorrhage II (ATACH II) was designed to determine the efficacy of rapidly lowering systolic BP in patients at an earlier time window after symptom onset than evaluated in previous trials. ${ }^{3}$

\section{POPULATION STUDIED}

This study enrolled adult patients who presented with spontaneous supratentorial intracranial hemorrhage who had at least one reading of systolic BP of $180 \mathrm{~mm} \mathrm{Hg}$ or more between symptom onset and the initiation of treatment. They included patients who presented to emergency departments with the following characteristics: age 18 years or older, initial Glasgow Coma Scale (GCS) of 5 or more, initial head computed tomography (CT) showing intraparenchymal hematoma of less than $60 \mathrm{~cm}^{3}$, and initiation of treatment within 3 hours from onset of symptoms. The recruitment window was later extended to 4.5 hours based on recent data, which suggested that hematoma expansion occurrence was equally prevalent among patients who presented between 0 and 3 hours and those who presented between 3 and 4.5 hours after symptoms onset. ${ }^{4}$

\section{STUDY DESIGN}

This was a multicentre, international, randomized, controlled trial that consecutively enrolled patients

From the *Emergency Medicine Residency Program, Faculty of Medicine, McGill University, Montreal, QC; and Departments of tMedicine and ‡Emergency Medicine, McGill University Health Centre, Montréal, QC.

Correspondence to: Dr. Sufyan Alrahbi, McGill University Health Centre, 1001 Boulevard Décarie, Room CS16237.1, Montreal, QC H4A 3J1; Email: Sufyan.alrahbi@mail.mcgill.ca 
presented to 110 hospitals in the United States, Japan, China, Taiwan, South Korea, and Germany. A total of 1,000 participants were recruited with a mean baseline systolic BP of $200.6 \pm 27.0 \mathrm{~mm} \mathrm{Hg}, 500$ were assigned to intensive treatment, and 500 were assigned to standard treatment. The initially planned sample size was 1,280 participants. However, enrolment was stopped early after 1,000 participants following a pre-specified interim analysis, which indicated futility.

\section{INTERVENTION}

Patients were assigned to the intensive treatment group (target systolic BP $110-139 \mathrm{~mm} \mathrm{Hg}$ ) and the standard treatment group (target systolic BP 140-179 mm Hg). The first-line treatment was intravenous nicardipine at a starting dose of $5 \mathrm{mg}$ per hour, titrated up by $2.5 \mathrm{mg}$ per hour every 15 minutes until the target BP was achieved. A pre-specified additional medication (intravenous labetalol) was used if the maximum dose of intravenous nicardipine $(15 \mathrm{mg}$ per hour) for 30 minutes failed to achieve the target BP. Intravenous diltiazem or urapidil can be used if labetalol was not available.

\section{OUTCOME MEASURED}

The primary outcome measured in this study was death or disability (as defined by modified Rankin scale of 4 to 6) at 90 days from randomization. Secondary outcomes include quality of life at 90 days as measured by EQ-5D utility index and visual analogue scale and hematoma expansion (defined as the increase from baseline hematoma volume of $33 \%$ or more) at 24 hours post-randomization using serial CT scans. In addition, safety outcomes were also recorded (defined by neurological deterioration of two or more points in GCS or increase of four or more points in the National Institutes of Health Stroke Scale), as well as serious adverse events within 72 hours after randomization considered to be related to treatment.

\section{RESULTS}

The demographic and clinical characteristics of the trial participants at baseline were similar in both groups. The mean interval between symptom onset and randomization was similar in both groups; however, BP target failure was higher in the intensive treatment group. Primary treatment failure occurred in 61 patients $(12.2 \%)$ in the intensive-treatment group versus $4(0.8 \%)$ in the standard-treatment group $(p<0.001)$. Secondary treatment failure occurred in 78 patients $(15.6 \%)$ in the intensive-treatment group versus $7(1.4 \%)$ in the standardtreatment group $(p<0.001)$. Among 961 participants in whom the primary outcome was reported, death or disability was observed in 186 participants $(38.7 \%)$ in the intensive-treatment group and in $181(37.7 \%)$ in the standard-treatment group with a measured relative risk of 1.04 (95\% confidence interval [CI] 0.85 to 1.27 ). There was no significant between-group difference in the ordinal distribution of the modified Rankin scale score at 3 months. In addition, neither the EQ-5D measures nor the percentages of patients with hematoma expansion differed significantly between the treatment groups. The percentage of patients with treatmentrelated, serious adverse events within 72 hours after randomization was $1.6 \%$ in the intensive-treatment group and $1.2 \%$ in the standard-treatment group. However, the percentage of patients with any serious adverse event during the 3 months after randomization was higher in the intensive-treatment group than in the standard-treatment group ( $25.6 \%$ v. $20.0 \%$; adjusted relative risk, $1.30 ; 95 \%$ CI 1.00 to $1.69 ; p=0.05$ ).

\section{STUDY CONCLUSION}

The treatment of participants with ICH to achieve a target systolic BP of 110 to $139 \mathrm{~mm} \mathrm{Hg}$ did not result in a lower rate of death or disability than standard reduction to a target of 140 to $179 \mathrm{~mm} \mathrm{Hg}$.

\section{COMMENTARY}

The ATACH II trial is a natural extension of the ATACH I trial, which confirmed the safety and tolerability of both regimen and goals of antihypertensive treatment in acutely hypertensive patients with $\mathrm{ICH}{ }^{5}$

This study was stopped after recruiting 1,000 participants of the planned enrolment target of 1,280 participants. The procedure of ending trials early can be problematic. If trials evaluating the benefit of an intervention are stopped prematurely, treatment effects can be overestimated because the imbalance between groups could equalize with additional time. Early cessation of a trial due to apparent futility may leave us in uncertainty as to whether the treatment is effective or harmful because a potential benefit might have been seen with increased patient recruitment. $^{6}$ 
In this study, the decision to stop the trial was based on pre-specified interim analyses, which would indicate treatment futility. This trial was powered to detect an effect difference of at least $10 \%$, and it was evident in the second planned interim analysis that this statistical threshold could not be achieved if enrolment continued and the trial was subsequently stopped. This is an acceptable approach in epidemiology to avoid the recruitment of additional subjects and giving them an ineffective therapy, particularly if there are adverse effects. ${ }^{7}$ This also allows for the efficient use of research resources (funding, participants, and time) because continuation of such studies represents suboptimal use of limited resources. ${ }^{8}$

A methodological issue impacting the result is the extension of the recruitment window from 3 to 4.5 hours following the onset of symptoms during the trial. This decision was based on a recent ATACH I study analysis performed by the same investigators of this trial, which demonstrated a similar incidence of hematoma expansion in those who presented between zero and 3 hours after onset of symptoms and those who presented between 3 and 4.5 hours. A breakdown of the exact number of participants recruited based on the original protocol and the extended time window is provided in the appendix of the trial, which showed that two thirds $(73.4 \%$ in intensive-treatment group and $68.2 \%$ in standardtreatment group) of those recruited were still included within 3 hours from the onset of symptoms.

It would have been useful to know at what exact point through the course of the trial was the recruitment window extended. An estimate of the number of excluded patients presenting within 3 to 4.5 hours from onset of symptoms before the extension in recruitment period may provide important additional information.

Although baseline characteristics of the study population appeared to be similar in both groups, the trial included only patients with mild to moderate supratentorial ICH. This is not representative of patients with large ICH, intracranial pressure elevation, and compromised cerebral perfusion pressure in whom intensive BP lowering is a concern for precipitating global or regional cerebral hypoperfusion. However, there may be a subset of patients who may benefit from intensive BP lowering as demonstrated with a recent retrospective study in which anticoagulation reversal and targeting $\mathrm{BP}$ lower than $160 \mathrm{~mm} \mathrm{Hg}$ was associated with a reduction in hematoma expansion in anticoagulation-associated $\mathrm{ICH}^{9}{ }^{9}$ Furthermore, more than half of the study participants had baseline
GCS of 15, which may indicate low severity of stroke and hence predict in itself better mortality outcome. ${ }^{10}$

Another point of concern is the disproportionately higher treatment failure in the intensive treatment group compared to the standard treatment group that may have diluted the effect of intensive treatment. This difference was observed in both the primary treatment failure $(12.2 \%$ v. $0.8 \%)$ and secondary treatment failure (15.6\% v. $1.4 \%)$. Perhaps a per-protocol analysis of patients who reached and maintained the targeted BP may provide a more precise reflection of treatment effect on mortality rates.

This study was conducted across 110 sites in 6 different countries. The standardization of monitoring and intensity of medical care provided at each site and low rates of withdrawal of care among subjects recruited in the study resulted in better outcome compared to previous studies. The observed rate of death or disability at 3 months in this trial $(37.7 \%)$ was significantly lower than that measured in previous literature (60\%). This may be explained by the majority of milder strokes (GCS of 15) included in this study. Additionally, the standardization of the drug treatment (intravenous nicardipine as the main agent) gave more homogeneous data compared to the INTERACT2 trial in which multiple drugs were used, resulting in subgroup analysis being uninformative.

\section{CONCLUSION}

The study suggests that there is no clear clinical advantage of intensively lowering $\mathrm{BP}$ below $140 \mathrm{~mm} \mathrm{Hg}$ compared to the guideline-recommended BP treatment (target BP less than $180 \mathrm{~mm} \mathrm{Hg}$ ) in patients with acute ICH and GCS of 15 , but methodological issues prevent definitive conclusions for more severe strokes. Furthermore, even in the context of a controlled trial setting achieving the intensive targeted $\mathrm{BP}$ was challenging and was also associated with some renal-related harm. This unfortunately still leaves us wondering about the ideal $\mathrm{BP}$ range to target in the emergency department for stroke patients with various severity clinical profiles.

Competing interests: None declared.

\section{REFERENCES}

1. Qureshi AI. The importance of acute hypertensive response in ICH. Stroke 2013;44:S67-9, doi:10.1161/ STROKEAHA.111.000758. 
2. Anderson CS, Huang Y, Arima H, et al. Effects of early intensive blood pressure-lowering treatment on the growth of hematoma and perihematomal edema in acute intracerebral hemorrhage: the Intensive Blood Pressure Reduction in Acute Cerebral Haemorrhage Trial (INTERACT). Stroke 2010;41(2):307-12.

3. Anderson CS, Heeley E, Huang Y, et al. Rapid bloodpressure lowering in patients with acute intracerebral hemorrhage. N Engl 7 Med 2013;368(25):2355-65.

4. Qureshi A, Palesch Y, ATACH II Investigators. Expansion of recruitment time window in Antihypertensive Treatment of Acute Cerebral Hemorrhage (ATACH) II Trial. 7 Vasc Interv Neurol 2012;5:6-9.

5. Qureshi AI, Antihypertensive Treatment of Acute Cerebral Hemorrhage Investigators. Antihypertensive treatment of acute cerebral hemorrhage (ATACH). Neurocrit Care 2007; 6(1):56-66.
6. Guyatt GH, Briel M, Glasziou P, et al. Problems of stopping trials early. BM7 2012;344:e3863.

7. Jitlal M, Khan I, Lee SM, et al. Stopping clinical trials early for futility: retrospective analysis of several randomised clinical studies. Br $\mathcal{F}$ Cancer 2012;107(6):910-7.

8. Schoenfeld DA, Meade MO. Pro/con clinical debate: it is acceptable to stop large multicentre randomized controlled trials at interim analysis for futility. Crit Care 2005;9(1):34-6, doi: $10.1186 / \operatorname{cc} 3013$.

9. Kuramatsu JB, Gerner ST, Schellinger PD, et al. Anticoagulant reversal, blood pressure levels, and anticoagulant resumption in patients with anticoagulation-related intracerebral hemorrhage. FAMA 2015;313(8):824-36, doi:10.1001/jama.2015.0846.

10. Parry-Jones AR, Abid KA, Di Napoli M, et al. Accuracy and clinical usefulness of intracerebral hemorrhage grading scores: a direct comparison in a UK population. Stroke 2013;44(7):1840-5. 